Theresa Schön

\title{
Symposiumsbericht
}

Knotenpunkt Botany Bay: Sozioökonomische Verflechtungen von Amerikanern, Briten und Franzosen in Australien, 17881800

\section{Symposium Review}

"Enlightened Powers: American, French \& British Interactions in Botany Bay, 1788-1800,"1

Ausgangspunkt der Überlegungen des von Therese-Marie Meyer (Martin-Luther-Universität Halle-Wittenberg) für die Gesellschaft für Australienstudien (GASt) veranstalteten und organisierten, internationalen und interdisziplinären Symposiums "Enlightened Powers: American, French \& British Interactions in Botany Bay, 1788-1800" war die Feststellung weitreichender Verbindungen (US-) amerikanischer, britischer und französischer Interessen im Zuge der Gründung der australischen Kolonien sowie der grundlegende Einfluss aufklärerischer Ideen und Werte auf das Leben in den ersten Siedlungen der terra australis. Das erklärte Ziel der Veranstaltung bestand in der mikro- und makrohistorischen Ausleuchtung dieser bisher von der Forschung wenig beachteten wissenschaftlichen, politischen, ökonomischen und militärischen Interaktionen. Das Symposium fand im Herbst 2014 in den Räumlichkeiten des Interdisziplinären Zentrums für die Erforschung der Europäischen Aufklärung (IZEA) der Martin-Luther-Universität Halle-Wittenberg in Halle an der Saale statt. Ermöglicht wurde die Veranstaltung durch die großzügige Unterstützung der Hamburger Stiftung zur Förderung von Wissenschaft und Kultur sowie das Mühlenberg-Zentrum für Amerikastudien.

\footnotetext{
1 Das Symposium wurde am 27. September 2014 an der Martin-LutherUniversität Halle-Wittenberg abgehalten.
} 
Mit der US-amerikanischen Unabhängigkeit in den Fokus der Europäer und Amerikaner gerückt, stellten die australischen Kolonien, zuvorderst die ersten Siedlungen in der Bucht von Sydney (Port Jackson) sowie Botany Bay, zentrale Orte der Aufklärung dar, wie die Organisatorin in Anlehnung an Dorinda Outram und John Gascoigne nachweist. Botany Bay sei nicht nur der Schauplatz eines sozialen Experiments gewesen. Die Kolonie habe darüber hinaus in gesellschaftlicher und kultureller Anlage zentrale aufklärerische Werte wie Fleiß und Eifer (industry and diligence) reflektiert, wobei strategisches Denken Basis des Handelns war. Der Handel habe eine zentrale Rolle gespielt, nicht nur in seiner Funktion, Güter des täglichen Lebens auszutauschen, sondern darüber hinaus als Vermittler von Wissen - eines Wissens über die Beherrschung der natürlichen Umgebung, dem in einer unbekannten Gegend wie dem australischen Kontinent eine überwältigende Rolle zugekommen ist. Das Symposium bot Gelegenheit, die vielfältigen Verflechtungen von Amerikanern und Europäern aus der Perspektive von Historiographie, Literaturwissenschaft und Kulturwissenschaft mikrohistorisch anhand einzelner historischer Akteure sowie editionsgeschichtlich und wissenschaftsgeschichtlich auf der Basis diverser wissenschaftlicher Publikationen (Reiseberichte, botanische Illustrationen) zu untersuchen.

In ihrem Plenarvortrag "Revolution, Rum, and Maroonage: The 'Pernicious American Spirit' at Port Jackson" begab sich die renommierte australische Historikerin Cassandra Pybus (Sydney University) auf die Spuren der ersten australischen bushranger und rekonstruierte drei Verbindungslinien zwischen dem australischen Kontinent und der jungen amerikanischen Nation: die Amerikanische Revolution, den Handel mit Rum und die Praxis der maroonage. Zum Inbegriff des australischen Charakters erklärt, waren bushranger Sträflinge, die vor einem Leben in Sklaverei und Gefangenschaft in den australischen Busch entflohen und auf diese Weise den Übergang von England nach Australien meisterten. Die Referentin zeichnete in dezidiert mikrohistorischer Manier die Wege zweier bushranger nach, nämlich die der afroamerikanischen Sträflinge 
John 'Black' Caesar und John Randall. ${ }^{2}$ Entgegen dem allgemein verbreiteten Standpunkt, der bushranger sei ein spezifisch australisches und dort einzigartiges sozioökonomisches Phänomen gewesen, argumentiert Cassandra Pybus, dass dieser australische Mythos vielmehr im Sinne ihres Vortragstitels über nordamerikanische Wurzeln verfügt. Durch sein Verhalten knüpfte der bushranger an die in Nordamerika bereits traditionelle Praktik der maroonage an, bei der Sklaven in den Busch bzw. die Wildnis entflohen und dort mit Hilfe der Eingeborenen eigene Siedlungen (von Gesetzlosen) aufbauten. Rum, ein grundlegendes Handelsgut amerikanischen Ursprungs, war weiterhin so bedeutsam in der neuen Kolonie, dass es u. a. im Fall 'Caesar' als Kopfgeld eingesetzt wurde. Die amerikanische Revolution schließlich brachte (entlaufene) Sklaven mit Soldaten zusammen, förderte persönliche Bande durch gemeinsame Erfahrungen im Unabhängigkeitskrieg und ermöglichte es z. B. John Randall, sich in der australischen Kolonie - nicht zuletzt durch Patronage - eine angesehene soziale Position zu erarbeiten.

Einen editionsgeschichtlichen Zugang verfolgte Alison E. Martin (University of Reading) in ihrem Beitrag zu "The Business of Books and Botany Bay: Publication, Dissemination and Knowledge Exchange". Am Beispiel der Publikationsgeschichte von Arthur Phillips Voyage of Governor Phillip to Botany Bay with an Account of the Establishment of the Colonies of Port Jackson and Norfolk Island (1789) erläuterte die Referentin, wie sich im Publikationsbetrieb Wissenschaft, Politik und Handel verknüpften. Anhand der Untersuchung diverser paratextueller Materialien zeigte sie auf, wie britische und französische Buchhändler, Verleger und Übersetzer bewusst die Rezeption von Phillips botanischem Werk zu manipulieren suchten. So betonte das der britischen Edition vorangestellte Gedicht von Erasmus Darwin die Bedeutung des Werks für den britischen industriellen Fortschritt, das britische wissenschaftliche Genie und die britische koloniale Expansion und damit den Erfolg der britischen Siedlungsbemühungen in Botany Bay. Die französische Ausgabe von 1791 hingegen, herausgegeben

${ }^{2}$ Vgl. auch: Pybus, Cassandra (2006). Black Founders: The Unknown Story of Australia's First Black Settlers. Sydney: UNSW Press. 
von Buisson und übersetzt von Millin de Grandmaison, repositionierte das Werk durch massive Eingriffe in dessen paratextuelle Ausgestaltung als internationalen Text. Millin, so die Referentin, strich nicht nur die dezidiert britischen Paratexte (Gedicht und Vignette), sondern setzte den Text durch extensives Fußnotenmaterial auch in Bezug zu Buffons, d. h. zu einem explizit französischen Klassifikationssystem. Mit ihrem Vortrag legte die Referentin überzeugend dar, wie sich die wissenschaftliche Publikationspraxis des späten 18. Jahrhunderts einerseits als rigoros nationales Projekt verstand und sich andererseits gleichzeitig an der globalen Erzeugung und dem weltweit vernetzten Austausch von naturhistorischem Wissen beteiligte.

In ihrem Vortrag "Enlightened Powers and 'Nature's Children': A French Colonial Encounter in the Time of Bonaparte" beleuchtete die Historikerin Nicole Starbuck (University of Adelaide) die anthropologischen Beobachtungen von Naturhistorikern, die $1802 \mathrm{im}$ Rahmen der französischen Expedition unter der Leitung des Kapitäns Nicolas Baudin in Port Jackson landeten. Nach heutigem Forschungsstand war diese Expedition der zeitgenössische Beleg für - die Referentin argumentiert hier in Anlehnung an Claude Blanckaert - das in dieser Periode dringende Bedürfnis, die Kenntnisse über die menschliche Natur auszubauen. Unter den historischen Bedingungen einer post-revolutionären, frühnapoleonischen Situation näherten sich Baudin und sein Anthropologe François Péron, der den offiziellen Reisebericht nach Baudins Tod veröffentlichte, den Aborigines von Port Jackson in bewusst naturwissenschaftlichem Gestus. Zum Gegenstand ihrer Betrachtungen wurden sowohl die Aborigines selbst, mit denen sie nachhaltigen Kontakt hatten, als auch die Interaktion zwischen indigenen Völkern und den britischen Kolonisatoren, der die Franzosen in Botany Bay beiwohnten. Das Bild, das Baudin und Péron von den australischen Ureinwohnern entwarfen, ist außerordentlich idealisierend. Beeinflusst durch republikanische Ideale, warf Baudin die grundsätzliche Frage auf, ob sich Briten und Franzosen in ihren zivilisatorischen Bestrebungen besser auf die eigene Nation beschränken sollten. Für inn waren die Aborigines von 
Port Jackson nicht weniger wild als die schottischen Hochländer; Péron schätzte ihr Sozialverhalten höher ein als das der Tasmanier. Der Reisebericht (Voyage de decouverte aux terres Australes) stellt damit ein bahnbrechendes anthropologisches Werk dar, wegweisend im Bereich ethnographischer Feldforschung.

Mit den Wechselbedingungen von Politik und Wissenschaft, ebenfalls am Beispiel der Botanik, befasste sich die Historikerin Henriette von Holleuffer in ihrem Beitrag "The Transported Flowers of Botany Bay: Herbarium, Greenhouse, or Botanical Ark? - Early Representations of the Australian Flora in the Work of British and French Naturalists, Botanical Artists and Gardeners". Ausgehend von Judy Dysons These, die botanische Illustration repräsentiere einen kulturellen Text, der verwandte Wissensformen (z. B. soziales, psychologisches oder politisches Wissen) transportiert, untersuchte die Referentin die Kollaboration von britischen und französischen Botanikern der Jahrhundertwende bei dem Versuch, Wissen über die australische Flora zu produzieren, zu konservieren und zu kultivieren. Die botanischen Illustrationen reflektierten, so Henriette von Holleuffer im Rückblick, das Interesse von sowohl Wissenschaftlern als auch Amateuren am Unbekannten und Undefinierten. Die frühen Abbildungen der australischen Flora stellten dabei nicht so sehr ein taxonomisches Ereignis dar, sondern vielmehr eine emblematische Interpretation des Exotischen. In symbiotischer Verbindung verknüpften sich hier Bild und essayistische Beschreibung sowie das Wirken wissenschaftlicher wie sozialer Netzwerke. Botanische Forschung bildete damit einen Bereich, der trotz europäischem Kriegsgeschehen über politische Grenzen hinweg zugänglich blieb. Die Kultivierung australischer Pflanzen in Britannien und Frankreich diente laut der Referentin der Befriedigung wissenschaftlicher Neugierde, weniger ökologischen Belangen. Das australische 'virtuelle Gewächshaus' schließlich, mit dessen hypothetischer Gestalt die Referentin ihren Beitrag begann, erscheint im Ergebnis weniger als botanische 'Arche', sondern vielmehr als Experiment - ein Experiment, im Rahmen dessen Botaniker Wissen austauschten und als dessen treibende Kraft britischer Pragmatismus sowie französischer Ästhetizismus gelten kann. 
Einem kulturwissenschaftlichen Ansatz folgend, wendete sich der Vortrag "Revolutions, Religion, and the Castle Hill Rebellion 1804" der Organisatorin des Symposiums, Therese-Marie Meyer (MartinLuther-Universität Halle-Wittenberg), dezidiert gegen die unter australischen Historikern etablierte monokausale, säkulare Lektüre der Castle Hill Rebellion, die diese primär als Konsequenz der starken Position der United Irishmen unter den Strafgefangenen lesen. Stattdessen plädierte die Referentin für eine multikausale Betrachtung der Ereignisse und ihrer Folgen. Konkret beschrieb sie drei fundamentale Ursachen für die Rebellion der irischen Sträflinge: Erstens, die unmittelbaren (administrativen) Auswirkungen der Deportation; zweitens, das Justizsystem und dessen Verfehlungen in Botany Bay und, drittens, den Katholizismus. Beziehen sich die ersten beiden Punkte explizit auf die schiere Zahl der irischen Sträflinge und deren Anteil an der Gesamtbevölkerung in Port Jackson (ein Drittel) sowie die Aussetzung solcher juristischer Grundlagen wie des habeas corpus in der Kolonie, so stellt der dritte Punkt den derzeit unter australischen Historikern am wenigsten berücksichtigten Aspekt dar. Die Referentin hob hervor, dass die Rolle des Katholizismus als zentrale Kraft der Castle Hill Rebellion bislang erheblich unterschätzt worden ist. Nach Therese Meyer deportierten Briten eine spezifisch anglikanische (statt einer französisch geprägten, philosophischen) Version der Aufklärung nach Botany Bay. Damit erzeugten die Kolonialherren Systemzwänge und entsprechende Folgen. Wurden die irischen Katholiken beispielsweise durch den verpflichtenden Besuch von Gottesdiensten gezwungen, sich dem anglikanischen Ritus zu beugen, war es innen gleichzeitig durch die spezifische Form ihrer Glaubenspraxis doch möglich, ihre Religion weiterhin auszuüben. Der daraus resultierende Opferdiskurs, so das Forschungsergebnis der Referentin, zeigte sich u. a. in eindrucksvollen Erinnerungssymbolen wie William Davies' Ecce-homo-Statue.

Die das Symposium beschließende Podiumsdiskussion "Americans in the South Pacific" gab den drei DiskussionsteilnehmerInnen Jennifer L. Anderson (Stony Brook University, New York), Andrew O'Shaughnessy (Center for Jefferson Studies, Monticello, Virginia) 
und Cassandra Pybus (Sydney University) die Gelegenheit, ihre Thesen zum Thema vorzustellen und mit dem Publikum zu erörtern. Alle drei TeilnehmerInnen bestätigten das im Laufe der Veranstaltung wiederholt festgestellte Defizit der australischen Historiographie, sich in ihrer Betrachtung der Kolonialgeschichte des Kontinents zu sehr auf das Empire zu konzentrieren - ganz im Sinne eines makrohistorischen Zugangs. Die Beispiele der ReferentInnen zeigten die Verbindungen zwischen US-Amerikanern, Franzosen und Briten im Zuge der Kolonialisierung von Botany Bay exemplarisch. Jennifer L. Andersons Forschungsergebnisse belegen anhand des (nordamerikanischen) Walfangs, wie stark der amerikanische und der australische Kontinent geschichtlich miteinander verwoben waren. Sie fand nicht nur Quellenbelege für amerikanische Ureinwohner auf den Walfängern, die in Botany Bay landeten, sondern auch für Afroamerikaner, also eine insgesamt kosmopolitische Struktur der Mannschaften. Andrew O'Shaughnessy argumentierte, dass die Erfahrungen, die die Briten sowohl in ihren indischen als auch in den (US-)amerikanischen Kolonien sammeln konnten, prägend auf die Gestaltung der ersten australischen Siedlungen einwirkten, insbesondere sichtbar in ihrem Verzicht auf die Gewährung von (politischer) Autonomie. Cassandra Pybus merkte Interessantes an: Die historischen Quellen belegen, dass sich unter den Mitgliedern der legendären First Fleet ein amerikanischer Ureinwohner befand. Obwohl dies zwar heute gemeinhin bekannt sei, habe aber bisher niemand die Frage gestellt, wer genau dieser Indianer war. Weiterhin wurden Ansätze zur Erschließung dieses bisher vernachlässigten Forschungsbereichs diskutiert. Neben einer grundlegend mikrohistorischen Herangehensweise schien sich eine Annäherung über den Begriff und das Konzept des Raums (spatial exploration) anzubieten.

\section{Fazit}

Die Beiträge machen deutlich, wie eng die britische, französische und amerikanische Geschichte mit der historischen Entwicklung in den ersten Kolonien von Botany Bay verbunden sind. Diese Verbindungen finden sich beispielhaft im Bereich der Politik und 
Ökonomie. Eine Erforschung der wissenschafts-, konfessions- und publikationsgeschichtlichen sowie anthropologischen Entwicklungen ist, wie die Diskussion belegt, äußerst fruchtbar und bringt auf mikro- wie makrogeschichtlicher Ebene wichtige Einsichten in die Art und Weise, wie die historischen Akteure in und um Port Jackson interagierten. Vor diesem Hintergrund ist dem von der Organisatorin ausgemachten und von den Teilnehmern bestätigten Desiderat zuzustimmen. In diesem Kontext bleibt auch die Rolle aufklärerischer Ideen genauer $\mathrm{zu}$ bestimmen. Insbesondere die exemplarischen Einblicke in die Publikationshistorie und Wissenschaftsgeschichte (Botanik und Anthropologie) decken grundlegende Prämissen der europäischen Aufklärung auf, die im Zuge der Erschließung und Kolonialisierung nach Botany Bay exportiert wurden. Gleichzeitig aber auch scheint die juristischmilitärische Vorgehensweise der in Port Jackson Verantwortlichen einen vollständigen Bruch mit den moralisch-ethischen Überlegungen des 18. Jahrhunderts zu offenbaren. Weitere Forschungen und deren Ergebnisse würden helfen, dieses Bild zu vervollständigen. Eine Fortsetzung der Diskussion und die Veröffentlichung der Beiträge sind vorgesehen.

\section{Works Cited}

Pybus, Cassandra, 2006. Black Founders: The Unknown Story of Australia's First Black Settlers. Sydney: UNSW Press. 